\title{
Statistical detection of Josephson, Andreev, and single quasiparticle currents in scanning tunneling microscopy
}

\author{
Wonhee Ko $\odot,{ }^{1}$ Eugene F. Dumitrescu, ${ }^{2}$ and Petro Maksymovych ${ }^{1, *}$ \\ ${ }^{1}$ Center for Nanophase Materials Sciences, Oak Ridge National Laboratory, Oak Ridge, Tennessee 37831, USA \\ ${ }^{2}$ Computational Sciences and Engineering Division, Oak Ridge National Laboratory, Oak Ridge, Tennessee 37831, USA
}

(Received 3 December 2020; revised 5 May 2021; accepted 12 July 2021; published 14 September 2021)

\begin{abstract}
We present a method to identify distinct tunneling modes in a tunable superconducting tunnel junction composed of a superconducting tip and a sample in a scanning tunneling microscope. Combining the relative decay constant of tunneling current extracted from $I-V-z$ spectroscopy with its statistical analysis over the atomic disorders in the sample surface, we identified the crossover of dominant tunneling modes between single charge tunneling, Andreev reflection (AR), and Josephson tunneling with respect to the bias voltage at a measurement temperature nearly half of the critical temperature. The method enables one to determine the specific tunneling regime independently of the spectral shapes and to reveal intrinsic modulation of AR and Josephson current by disorder that will be crucial for superconducting quantum information processing.
\end{abstract}

DOI: 10.1103/PhysRevResearch.3.033248

\section{INTRODUCTION}

The tunnel junction between superconductors is the heart of modern quantum information devices. Superconductor qubits, which are some of the most promising scalable qubits right now, heavily rely on the Josephson effect, which is quantum tunneling of Cooper pairs between the two superconductors [1-3]. The superconducting tunnel junction can also probe the nature of superconductivity because tunneling behavior is highly dependent on the type of superconductors, such as either $s$ wave or $d$ wave [4,5] and trivial or topological [6].

In the superconducting tunnel junction, the interplay between electrons and Cooper pairs results in various modes of tunneling, i.e., single charge tunneling, Andreev reflection (AR), and Josephson tunneling [7]. The tunneling mechanisms were previously studied both experimentally and theoretically on various systems including planar devices [8-10], break junctions [11], and scanning tunneling microscopes (STMs) [12-17]. However, the previous studies mostly focused on the clean limit (absence of disorder) and the junction temperature much less than the critical temperature. It is less explored how tunneling mechanisms and their crossover behave in the presence of disorder and finite temperature, which is crucial for both practical application of superconducting tunnel junctions and fundamental investigation of proximity of phase transitions. These regimes require the development of a robust method to distinguish between tun-

\footnotetext{
*maksymovychp@ornl.gov

Published by the American Physical Society under the terms of the Creative Commons Attribution 4.0 International license. Further distribution of this work must maintain attribution to the author(s) and the published article's title, journal citation, and DOI.
}

neling mechanisms that is complementary to detailed analysis of spectral shapes.

STM is a fitting platform to study the behavior of various tunneling mechanisms with respect to the junction geometry since it can precisely control the tunneling distance down to picometer resolution and determine the atomic-scale geometry of the tunneling junction by imaging the surface. In this paper, we used STM with a superconducting tip to make a superconducting tunnel junction with a vacuum tunneling barrier that is controllable to picometer precision. The spectroscopy based on $I-V-z$ characteristics allowed us to extract the relative decay constant of the tunneling current with respect to the tip height and display the crossover between the single charge tunneling, AR, and Josephson tunneling. Moreover, the relative decay constant of different tunneling modes displays distinct spatial distribution relative to the atomic disorder, whose statistical analysis results in the identification of dominant tunneling mechanisms even at the measurement temperature as high as nearly half of the critical temperature. Meanwhile, tight-binding simulation shows the broad variability of tunneling probability of AR as a function of quasiparticle energy, whose resonant tunneling at the superconducting gap edge ultimately translates into an intermediate decay constant between single charge and Josephson tunneling. Our results indicate that the statistical analysis of $I-V-z$ spectroscopy is a powerful method to distinguish various tunneling regimes with minimal assumption for the physical model and specific spectral shapes.

\section{EXPERIMENTAL METHODS}

The experiment was performed in SPECS JT-STM operated at ultrahigh vacuum condition $\left(<10^{-10} \mathrm{mbar}\right)$ and base operating temperature of $1.2 \mathrm{~K}$. The surface of an $\mathrm{Pb}(110)$ single crystal was cleaned by repeating sputtering-annealing cycles several times $[16,18]$. The STM tip was coated with Pb 


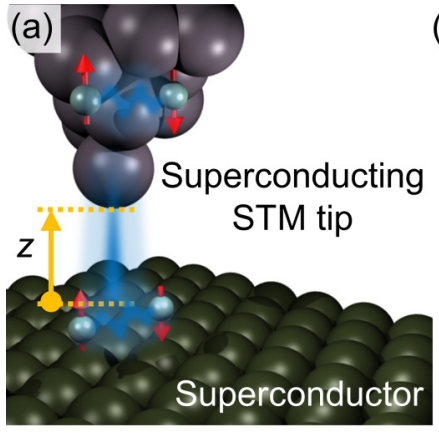

(b) Single quasiparticle tunneling $\left(e V>\Delta_{s}+\Delta_{t}\right)$ Tip

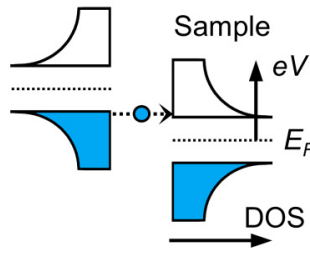

$I \propto T_{N} \propto e^{-\kappa_{N} Z}$

(c) Multiple $(m)$ Andreev reflection $\left(e V<\Delta_{s}+\Delta_{t}\right)$

(d) Josephson tunneling $(e V \sim 0)$
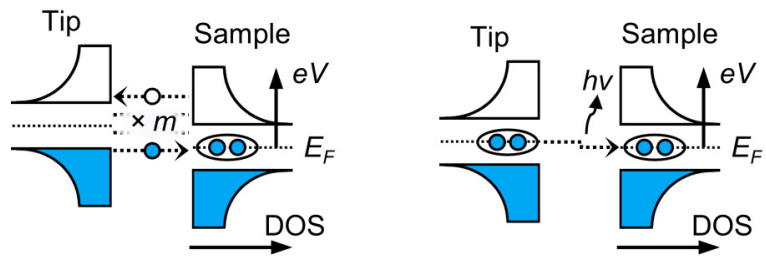

$I \propto T_{N} \cdot\left(T_{N}\right)^{m}$ $\propto e^{-(m+1) \cdot \kappa_{N} Z}$

than $\Delta_{s}+\Delta_{t}$, single charge tunneling is suppressed due to vanishing density of states. Therefore, the tunneling current becomes dominated by higher order processes such as single and multiple AR, wherein the apparent decay constants $\kappa$ deviate from $\kappa_{N}$ due to their higher order scaling to the tunneling probability $T_{N}$ [Fig. 1]. Cuevas et al. [23,24] showed that the tunneling probability of the multiple AR (MAR) process with $m$ times reflection is proportional to $\left(T_{N}\right)^{m+1}$ by using the Keldysh nonequilibrium Green function method, and so $\kappa$ of MAR becomes $(m+1) \cdot \kappa_{N}$ [Fig. 1(c)]. Meanwhile, Josephson tunneling in STM happens in the dynamical Coulomb regime, where a small capacitance of STM tunnel junction forces Cooper pairs to tunnel sequentially while emitting microwave photons [Fig. 1(d)] [15,16,25]. In this regime, an inelastic tunneling process adds another factor of $E_{J} / E_{C}$ to the tunneling probability, where $E_{J}$ is the Josephson energy, and $E_{C}$ is the capacitive charging energy [26]. The AmbegaokarBaratoff $(\mathrm{AB})$ formula shows that $E_{J}$ is proportional to the tunneling probability $T_{N}$ [8], while $E_{C}$ stays almost constant with subnanometer shift of $z$ because it mostly depends on the macroscopic tip geometry [15,27], so $\kappa$ becomes $2 \kappa_{N}$. Curiously, based on these observations, AR should be indistinguishable from Josephson tunneling, contrary to what we show below.

FIG. 1. (a) Schematic of tunable superconductor tunnel junction composed of a superconducting scanning tunneling microscope (STM) tip and a superconducting sample. (b)-(d) Schematic diagrams of single charge tunneling, multiple Andreev reflection, and Josephson tunneling, respectively. Under each diagram, the expected formula of exponentially decaying current is written for the asymptotic case of large $z$.

by applying a bias voltage of $100 \mathrm{~V}$ to the tungsten tip and then dipping it into the $\mathrm{Pb}(110)$ single crystal while limiting the maximum current to $100 \mu \mathrm{A}$ with a $10 \mathrm{M} \Omega$ resistor in series [19]. Conversely, $\mathrm{Pb}$ on the tip can be removed by field emission on $\mathrm{Au}(111)$ with a bias voltage of $100 \mathrm{~V}$. The $I-V$ curves were acquired by sweeping direct current bias without any additional electrical signals. Here, $d I / d V$ curves were obtained by numerical differentiating the $I-V$ curves and Gaussian smoothing.

\section{RESULTS}

\section{A. Tunneling mechanisms in a superconducting STM junction}

Figure 1(a) shows the schematic of the tunable superconducting tunnel junction realized by STM. Precise control of the tip height $z$ enables the measurement of the decay of tunneling current $I$ with respect to $z$. Theoretical models based on Bardeen's formalism [20] predicted that the single electron tunneling probability $T_{N}$ decays exponentially with $z$ in the asymptotic case of large $z$ [21], $I(z) \propto e^{-\kappa z}$, with the effective decay constant $\kappa$. In case of a superconducting tunnel junction with large $z$, single charge tunneling dominates over AR when the bias is larger than the sum of the sample superconducting gap $\Delta_{s}$ and the tip superconducting gap $\Delta_{t}$ [Fig. 1(b)] [10,12,22,23]. The decay constant of single charge tunneling is the same as the one in the normal state [20], which we denote as $\kappa_{N}$. However, when the bias is lower

\section{B. $I-V-z$ spectroscopy and relative decay constant}

Figure 2 shows the $I-V-z$ spectroscopy measurement on the $\mathrm{Pb}$ tip- $\mathrm{Pb}(110)$ junction [inset of Fig. 2(a)]. Figure 2(a) displays the $I-V$ curves while approaching the tip to the sample surface. Here, $d I / d V$ spectra from the numerical derivatives of $I$ - $V$ curves show a clear superconducting gap between the coherence peaks at $\pm 2.6 \mathrm{mV}$ [Fig. 2(b)], as expected from the $\Delta_{s} \approx 1.35 \mathrm{meV}$ in $\mathrm{Pb}$ and $\Delta_{t}$ slightly less than $\Delta_{s}[12,16]$. Inside the superconducting gap, the structure of $d I / d V$ first rapidly decreases with approaching the Fermi level, but also reveals an increase of tunneling conductance at the Fermi level, particularly for spectra acquired with closest proximity between the tip and the surface. The bias lower than the superconducting gap indicates that the origin of the intragap features is AR or Josephson tunneling, or perhaps both. We note that the feature at the Fermi level is broader than the previous reports of Josephson currents in the STM junction $[12,17]$, possibly due to the effective junction temperature of $3.2 \mathrm{~K}$ that is slightly higher than the base temperature (see Appendix A and Fig. 5).

The decay constant $\kappa\left(V_{b}\right)$ was extracted by fitting $I-z$ or $d I / d V-z$ curves to the exponential function (see Appendix B and Fig. 6). We further defined the relative decay constant as $\kappa / \kappa_{N}$, where $\kappa_{N} \equiv \kappa\left(V_{\max }\right)$. In Fig. 2(c), we plot $\kappa / \kappa_{N}$ extracted from $I-z$ curves of the $\mathrm{Pb}$ tip- $\mathrm{Pb}(110)$ junction. It is clearly shown that $\kappa / \kappa_{N}=1$ for $e\left|V_{b}\right|>\Delta_{s}+\Delta_{t}$, as expected for single charge tunneling, but as $e\left|V_{b}\right|$ becomes smaller than $\Delta_{s}+\Delta_{t}$, it increases from 1 to almost exactly 2 around $V_{b}=0 \mathrm{mV}$, as expected for Josephson tunneling $[15,26]$. We further confirmed that the origin of the current at $\sim 0 \mathrm{meV}$ is Josephson tunneling by fitting $I-V$ curves with $P(E)$ theory and found a perfect match of the Josephson critical current to the AB formula (see Appendix C and Fig. 7) [8,15]. The spike 


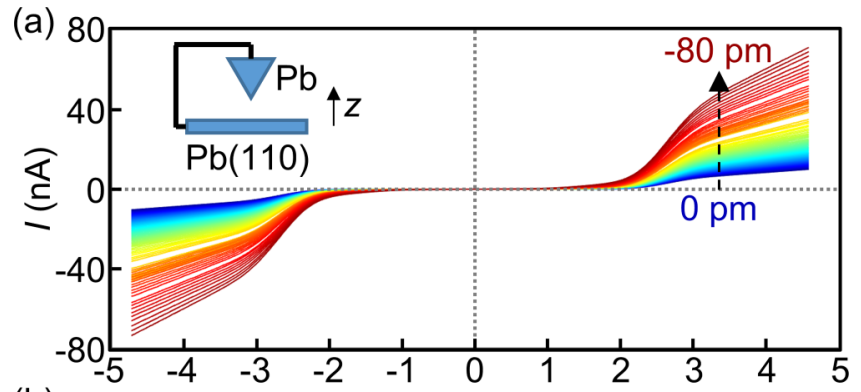

(b)
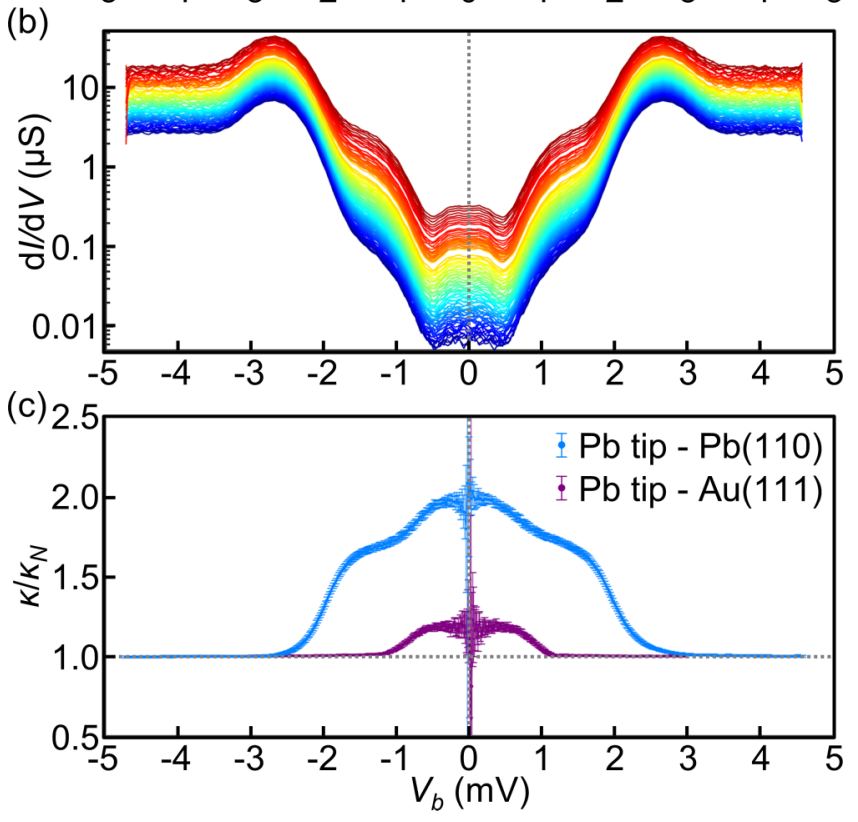

FIG. 2. (a) $I-V$ curves between $\mathrm{Pb}$ tip and $\mathrm{Pb}(110)$ for every $1 \mathrm{pm}$ step as the tip approaches from 0 to $-80 \mathrm{pm}$. $z=0$ is defined as the tip height at $V_{b}=4.6 \mathrm{mV}$ and $I=10 \mathrm{nA}$. (b) $d I / d V$ curves obtained by numerical differentiation of $I-V$ curves in (a). (c) Relative decay constant $\kappa / \kappa_{N}$ for $\mathrm{Pb}$ tip on $\mathrm{Pb}(110)$ and $\mathrm{Au}(111)$.

feature right at $0 \mathrm{mV}$ is an artifact due to very small currents and the resulting uncertainty in the fitting of the $I-z$ curves.

The transition between $\kappa / \kappa_{N}=1$ and $\kappa / \kappa_{N}=2$ proceeds through two nearly flat plateaus at around $\left|V_{b}\right|=1.0-1.5 \mathrm{mV}$ with $\kappa / \kappa_{N}=1.7 \pm 0.05$. The expected bias for single AR is $e\left|V_{b}\right| \sim \Delta_{s} \approx 1.35 \mathrm{meV}$ [16], so we can tentatively assign the plateaus in $\kappa / \kappa_{N}$ accordingly. However, $\kappa / \kappa_{N}=1.7$ for single AR deviates from the expected value of 2 [23,24], and so in the following sections, we further confirm that this value is from single AR and explore the origin of the deviation. We note that a control measurement on the $\mathrm{Pb}$ tip- $\mathrm{Au}(111)$ junction displayed $\kappa / \kappa_{N}=1$ outside the superconducting gap but increased to 1.2 inside the gap. The observation qualitatively supports our assignment of $1<\kappa / \kappa_{N}<2$ for single $\mathrm{AR}$ and that the double step feature in the $\mathrm{Pb}$ tip- $\mathrm{Pb}(110)$ junction is not an artifact of the $\mathrm{Pb}$-coated tip. We attribute the main difference in the value of $\kappa / \kappa_{N}$ for single AR of $\mathrm{Pb}$ tip- $\mathrm{Pb}(110)$ and $\mathrm{Pb}$ tip- $\mathrm{Au}(110)$ to thermal broadening effects, as discussed in the latter part of this paper. A structural contribution due to the difference of the chemical compositions of the tip and the surface may also play a role in determining the specific values for AR due to difference in
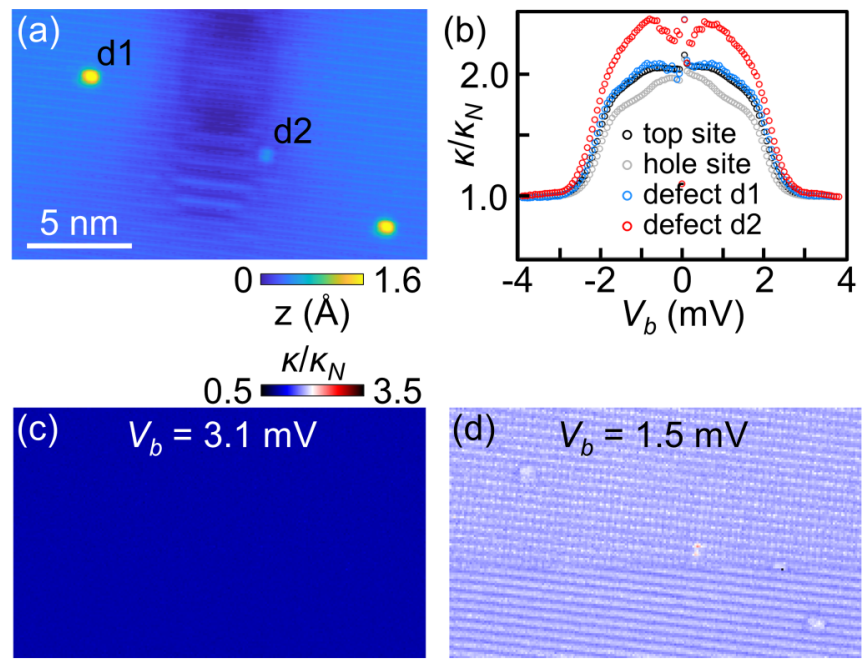

(e) $V_{b}=0.79 \mathrm{mV}$

(f) $\quad V_{b}=0.24 \mathrm{mV}$
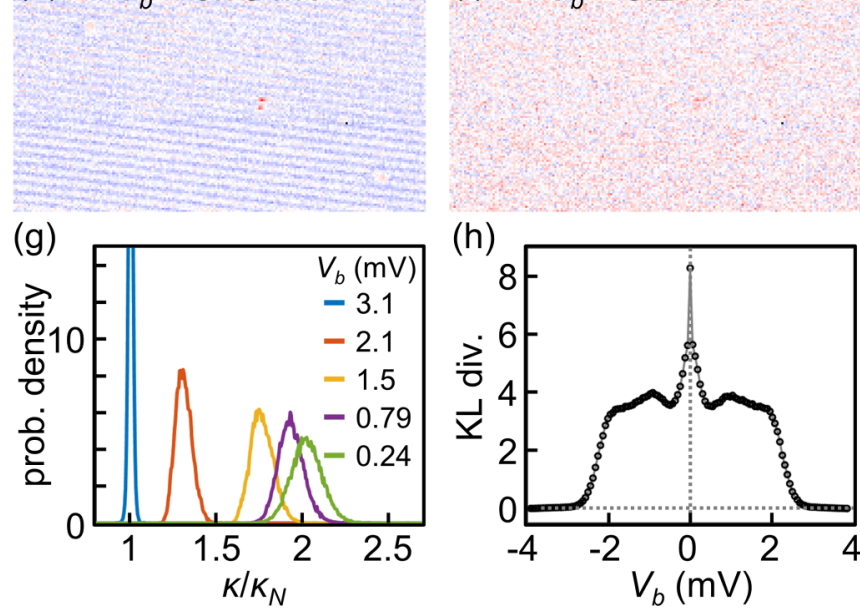

FIG. 3. (a) Scanning tunneling microscope (STM) topograph of $\mathrm{Pb}(110)\left(V_{b}=4.6 \mathrm{mV}, I=10 \mathrm{nA}\right)$. (b) $\kappa / \kappa_{N}$ curves of four different types of sites in (a). (c)-(f) The maps of the relative decay constant at the biases labeled on top. (g) The probability density from the histogram of the $\kappa / \kappa_{N}$ values in the map at certain bias. (h) KullbackLeibler (KL) divergence between the probability density of $\kappa / \kappa_{N}$ values at maximum bias and other biases, centered on the mean value.

the orbital configurations [11] and possible finite size effects on superconducting properties of the STM tip.

\section{Statistical analysis of relative decay constant over disorders}

To gain complementary insight into the crossover of tunneling mechanisms for different $\kappa / \kappa_{N}$ regimes, we observe the distinct behavior of AR and Josephson tunneling with respect to disorder. It is known that $A R$ strongly depends on the detailed atomic structure of the junction [28,29], while Josephson tunneling does not because Cooper pairs are delocalized over the coherence length $(\sim 80 \mathrm{~nm}$ in $\mathrm{Pb})$ [30]. Therefore, they should be discernable via spatial distribution in heterogeneous samples.

To probe the feasibility of differentiating the mechanisms via disorder, we acquired the distance-dependent spectroscopy in a wide area of the $\mathrm{Pb}(110)$ surface with a natural population of defects [Fig. 3(a)]. The STM topography 
displays two types of surface defects, labeled $d 1$ and $d 2$, and one subsurface defect that generates a scattering pattern around the center. Figure 3(b) shows four representative $\kappa / \kappa_{N}$ curves: taken on top of the surface $\mathrm{Pb}$ atoms (top site), on the hole between the surface $\mathrm{Pb}$ atoms (hole site), and on defects $d 1$ and $d 2$. For $\left|V_{b}\right|=0.5-2 \mathrm{mV}$, where MAR dominates, there is large variation of $\kappa / \kappa_{N}$. Even on the flat surface, the single $\mathrm{AR}$ at $\left|V_{b}\right|=1.3 \mathrm{meV}$ exhibits atomic-scale variation, where the $\kappa / \kappa_{N}$ of the hole site and top site varies from 1.7 to 1.9. Defects display more vivid contrast, as demonstrated in the $\kappa / \kappa_{N}$ of $d 2$, which rises to 2.5 at $\left|V_{b}\right|=0.7 \mathrm{mV}$. Here, $\kappa / \kappa_{N}>2$ indicates that the atomic structure of the defect facilitates the second AR. Such an enhancement of higher order AR highly depends on the atomic structure of the defects, as demonstrated in the $\kappa / \kappa_{N}$ of defect $d 1$, whose value is $<2$ for all bias. However, as $V_{b}$ approaches $0 \mathrm{mV}, \kappa / \kappa_{N}$ in all positions converges to 2 , whose spatial uniformity supports the transition from AR to Josephson tunneling.

For statistical analysis of the effect of disorder, we then acquired $I-V-z$ curves on the regularly spaced grid. The maps of $\kappa / \kappa_{N}$ at specific bias in Figs. 3(c)-3(f) and their corresponding histograms in Fig. 3(g) confirm the observations from the individual spectra: for single charge tunneling, the map is very uniform with the values $\sim 1$ [Fig. 3(c)]; in the regime of MAR, $\kappa / \kappa_{N}$ displays atomic-scale variation that follows the lattice of $\mathrm{Pb}(110)$ and atomic defects [Figs. 3(d) and 3(e)], giving rise to broad distribution with multiple peaks and a long tail in the histogram; and at $\sim 0 \mathrm{mV}$, the values of $\kappa / \kappa_{N}$ become more uniform [Fig. 3(f)], albeit with still relatively broad distribution (see the movie in the Supplemental Material [31] for the full dataset). Note that there was a tip change at $\sim \frac{2}{5}$ point in the $y$ axis of the $\kappa / \kappa_{N}$ maps, but the qualitative behavior of $\kappa / \kappa_{N}$ does not change.

The crossover of mechanisms can be effectively captured by calculating the Kullback-Leibler (KL) divergence between the probability densities of the $\kappa / \kappa_{N}$ values as a function of tunneling bias. Figure $3(\mathrm{~g})$ shows the probability density of $\kappa / \kappa_{N}$ at certain bias from the numerical approximation of $\kappa / \kappa_{N}$ histograms, and Fig. 3(h) is the KL divergence between the probability density at certain bias and the maximum bias, after centering them at the mean value [32]. The remarkably sharp transitions as a function of bias are evident. Most notably, the transition between AR and Josephson regime occurs at $\left|V_{b}\right| \sim 0.5 \mathrm{mV}$ in the KL divergence as a steep rise, which is also consistent with the bias where $\kappa / \kappa_{N}$ maps become uniform and lose atomic features [31]. We note that, in the calculation of the KL divergence, we intentionally subtracted the mean value of the distributions to focus the comparison on the variation rather than averaged value of the $\kappa / \kappa_{N}$. Thereby, the statistical analysis provides independent confirmation of our hypothesis of the distinct behavior of AR and Josephson current over disorder from the transition in $\kappa / \kappa_{N}$ distribution.

\section{Tight-binding simulation of AR}

To simulate AR as a function of basic properties of a tunnel junction, we utilized KWANT code for tight-binding transport calculations [33]. Following a basic algorithm, the Bogoliubov-de Gennes particle-hole symmetric Hamiltonian
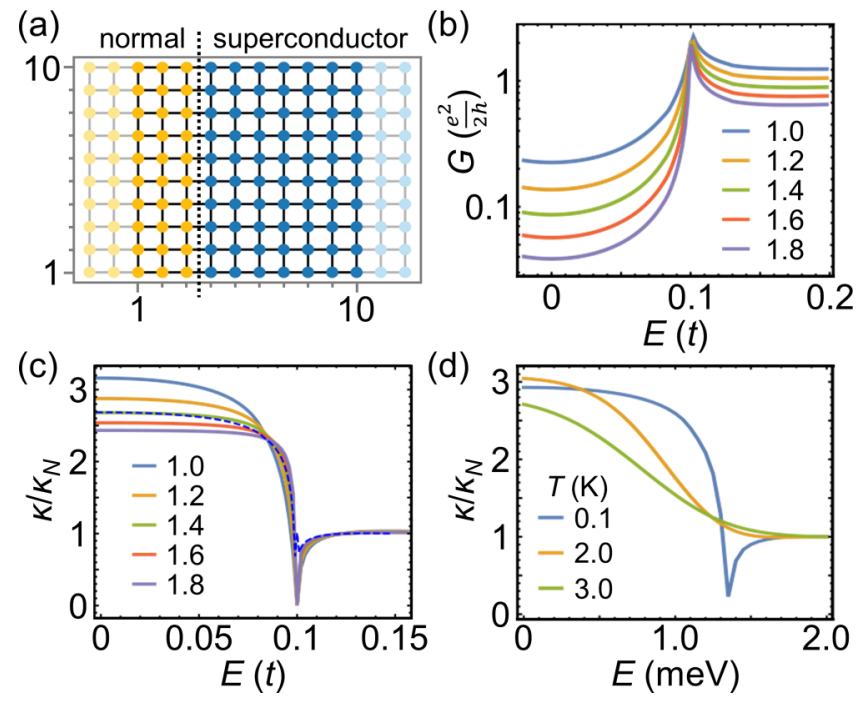

FIG. 4. (a) Schematic of the lattice structure of normalsuperconductor junction for transport conductance simulation. (b) The conductance vs energy for different barrier heights. The superconducting gap was chosen as $0.1 t$, where $t$ is the hopping integral. (c) The relative decay constant for different barrier heights (shown in legends, in units of $t$ ). (d) The relative decay constant graphs with increasing thermal broadening $\left(\Delta_{s}=0.1 t=1.35 \mathrm{meV}\right.$ for comparison with the experiment).

was implemented in a spinless system without magnetic field on a square lattice. The system is schematically shown in Fig. 4(a). The scattering region is localized between columns 3 and 4, with the superconducting gap being nonzero in columns 4-10 (blue dots) and the normal lead in columns 1-3 (yellow dots). The shaded dots on each side denote the beginning of infinite leads attached to each region, with the same corresponding Hamiltonians as in the regions. The conductance $G$ calculated for such geometry clearly exhibits a superconducting gap and resonant AR on the gap edge [Fig. 4(b)]. Subsequently, we systematically varied the properties of the tunneling barrier to calculate conductance as a function of barrier properties. The analysis of the apparent decay constants was then carried out similarly to the experiment.

As seen in Fig. 4(c), the $\kappa / \kappa_{N}$ exhibits a broad range, from $\sim 0$ to $\sim 3$, across the superconducting gap. The values $\sim 0$ originate from resonant Andreev tunneling at the gap edge [28], where the transmission probability is enhanced, as is common for generic resonant tunneling. The effect of the resonance decays as the energy decreases toward the middle of the gap. Eventually, $\kappa / \kappa_{N}$ exceeds 2 and reaches values as high as 3. The results for AR are similar for the cases where we tune the barrier height and barrier width, although the resonance effect is most pronounced in the barrier-heightdependent calculation. It is also important to consider the effect of broadening on these results, which were calculated at $0 \mathrm{~K}$. A thermal broadening of conductance applied to the calculated $\kappa / \kappa_{N}$ reveals that the resonance-mediated decrease $<1$ is quickly smeared $>2 \mathrm{~K}$ [Fig. 4(d)]. However, the effect of the resonance is still present, producing intermediate $\kappa / \kappa_{N}$ between 1 and 2, over the width of about half the superconducting gap. Overall, these results are qualitatively like the experimental observations in Fig. 2(c). 
Both $>2$ and $<2$ values for $\kappa / \kappa_{N}$ in the Andreev regime allow us to propose a simple conceptual picture of the crossover of tunneling mechanisms as three parallel channels whose resistance depends on tip height $z$ and the tunneling energy $e\left|V_{b}\right|$. When $e\left|V_{b}\right|>\Delta_{s}+\Delta_{t}$ and the tunneling barrier is large, single charge tunneling is dominant, even though the AR and Josephson tunneling is possible [22,23]. Meanwhile, in the region of the superconducting gaps, the preponderance of Josephson vs Andreev tunneling will be determined by their decay constant, with the smaller decay constant determining the dominant current. Just below the gap, the resonance effect favors single AR. Toward zero bias, higher order MARs appear successively, but also Josephson tunneling starts to compete with MAR, and eventually, crossover happens because MAR has a higher decay constant than Josephson tunneling. The crossover will be sensitive to many specifics of the tunneling junction and the measurement conditions. For example, the role of prefactors in the exponential decay of tunneling current remains to be understood in future analysis. Conversely, detailed understanding of the crossover is likely to provide a window into the properties of impurities in superconductors.

\section{CONCLUSIONS}

In summary, we combined the $I-V-z$ spectroscopy of the STM-based superconducting tunnel junction with the statistical analysis over atomic-scale disorder to provide the clear illustration of crossovers between single charge tunneling, MAR, and Josephson tunneling. The analysis enabled determination of the specific tunneling regime independently of the spectral shapes, which provides a valuable complement to other methods of analysis of superconducting junctions. Rigorously comparing and correlating the effects of disorder will deepen our understanding on the nature of superconductivity. For example, revealing intrinsic modulations of the AR and Josephson currents would provide a pathway to identify pairing symmetry [13], inhomogeneity of superfluid density $[17,34,35]$, and possible existence of exotic quasiparticles $[36,37]$ at the heart of modern quest for quantum and topological computing.

The DOE will provide public access to these results of federally sponsored research in accordance with the DOE Public Access Plan [38].

\section{ACKNOWLEDGMENTS}

We gratefully acknowledge Stephen Jesse for support and discussion of this paper. This research was conducted at the Center for Nanophase Materials Sciences, which is a U.S. Department of Energy (DOE) Office of Science User Facility. A portion of this research (WK, PM) was sponsored by the Laboratory Directed Research and Development Program (as a part of the Quantum Initiative) of Oak Ridge National Laboratory, managed by UT-Battelle, LLC, for the DOE. Part of the work (ED) was sponsored by the DOE, Office of Science, Basic Energy Sciences, Materials Sciences and Engineering Division. This paper has been partially supported by UTBattelle, LLC, under Contract No. DE-AC0500OR22725 with

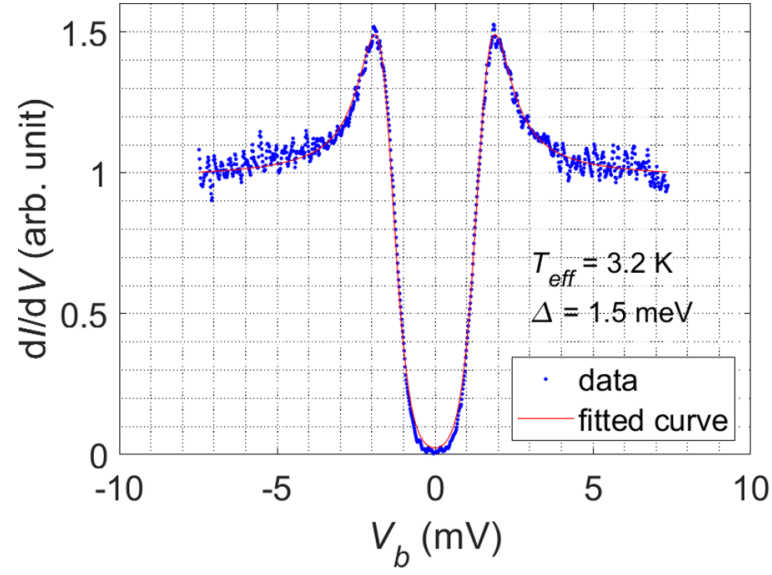

FIG. 5. $d I / d V$ spectra of $\mathrm{Pb}(110)$ measured by normal metal tip (blue dots) and fitting of the curve with the Dynes formula (red line).

the DOE. The U.S. Government retains, and the publisher, by accepting the article for publication, acknowledges that the U.S. Government retains a nonexclusive, paid-up, irrevocable, worldwide license to publish or reproduce the published form of this paper or allow others to do so for U.S. Government purposes.

The authors declare no competing interests.

\section{APPENDIX A: EFFECTIVE ELECTRON TEMPERATURE OF THE STM TUNNEL JUNCTION FROM THE GAP FITTING}

To estimate the effective temperature of the STM tunnel junction, we measured the superconducting gap of $\mathrm{Pb}(110)$ with a normal metal tip and fit the curve with the Dynes formula:

$$
\begin{aligned}
\rho_{\mathrm{BCS}}(E) & =\left\{\begin{array}{ll}
0 & \text { if }|E|<\Delta \\
\operatorname{Re}\left\{\frac{E-i \Gamma}{\left[(E-i \Gamma)^{2}-\Delta^{2}\right]^{1 / 2}}\right\} & \text { if }|E|>\Delta,
\end{array},\right. \\
\rho\left(E, T_{\text {eff }}\right) & =\int \rho_{\mathrm{BCS}}(E) f^{\prime}\left(E, T_{\text {eff }}\right) d E,
\end{aligned}
$$

where $\rho$ is the electron density of states, $\Delta$ is the superconducting gap, $\Gamma$ is the intrinsic broadening, $T_{\text {eff }}$ is the effective electron temperature, and $f(E, T)$ is the Fermi-Dirac distribution given as

$$
f(E, T)=\frac{1}{e^{E / k_{B} T}-1} .
$$

Figure 5 shows the result of fitting $d I / d V$ spectrum to $\rho\left(\mathrm{eV}, T_{\mathrm{eff}}\right)$, which gives $T_{\mathrm{eff}}=3.2 \mathrm{~K}, \Delta=1.5 \mathrm{meV}$, and $\Gamma=$ $4.7 \times 10^{-7} \mathrm{meV}$.

\section{APPENDIX B: EXTRACTION OF THE DECAY CONSTANTS FROM $I-z$ FITTING AND THEIR ERROR ANALYSIS}

To extract the decay constant $\kappa$ from the $I-V-z$ curves, we rearranged the data to plot $I-z$ or $d I / d V-z$ curves at different biases and fitted the curves to exponential curves. Figure 6(a) shows the examples of the $I-z$ curves and overlayed exponential fitting, and Fig. 6(b) shows $\kappa / \kappa_{N}$ extracted from the fitting and $R^{2}$ of the fit. Here, $R^{2}$ is $\sim 1$ for all bias ranges, except the very near zero, where the current reached the noise 

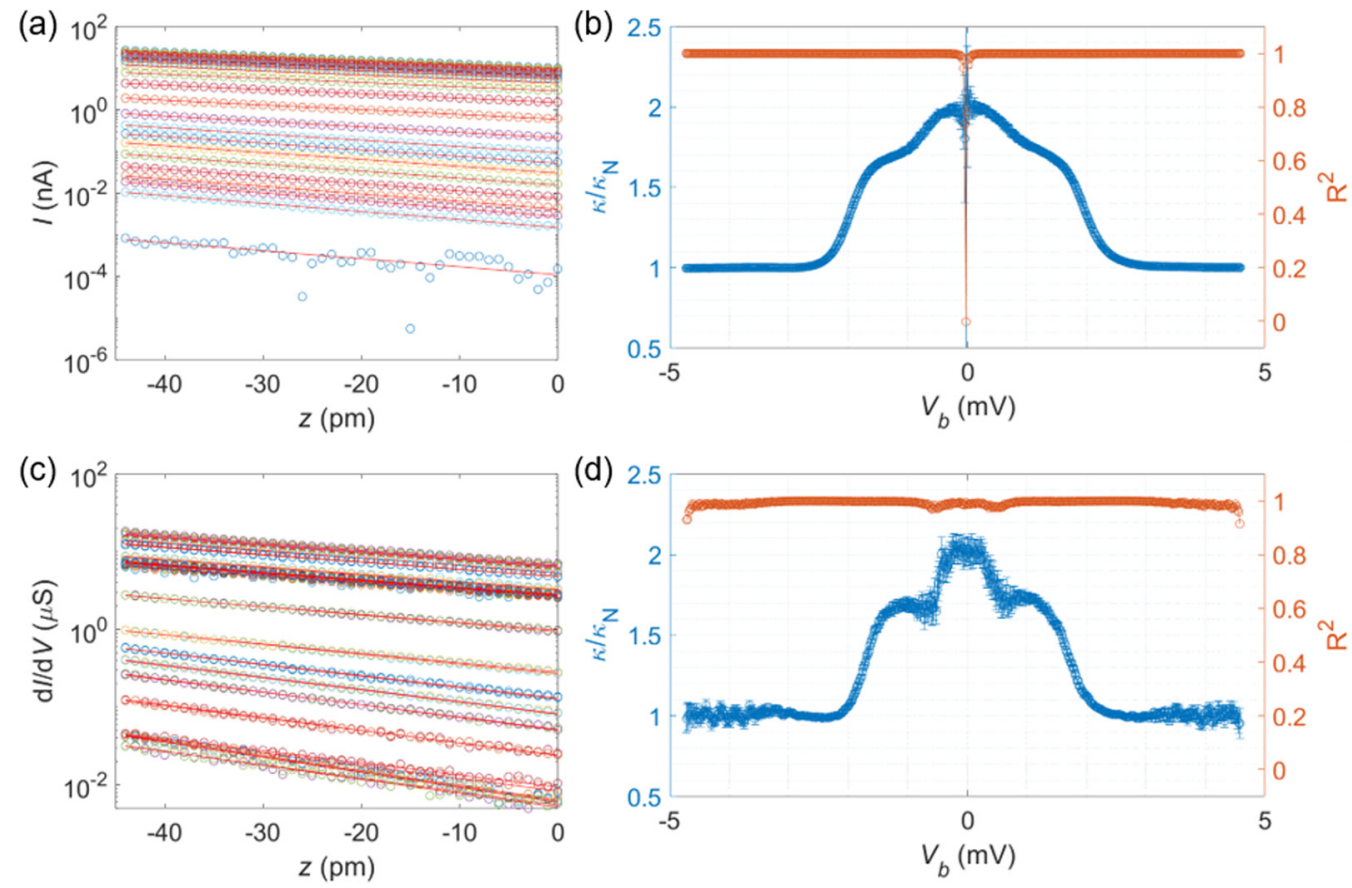

FIG. 6. (a) $I-z$ curves for different $V_{b}$ fitted to the exponential function (red lines). Note that the $y$ axis is in log scale. (b) The relative decay constant $\kappa / \kappa_{N}$ and the $R^{2}$ from the fitting in (a). (c) $d I / d V-z$ curves for different $V_{b}$ fitted to the exponential function (red lines). Note that the $y$ axis is in log scale. (d) The relative decay constant $\kappa / \kappa_{N}$ and the $R^{2}$ from the fitting in (c).

limit and has large fluctuation [see lowest curve in Fig. 6(a)]. The $R^{2}$ analysis shows the fitting is nearly perfect for most bias ranges, except the very near zero, which also explains the large error bar in $\kappa / \kappa_{N}$ at around the zero bias. Figures $6(\mathrm{c})$ and $6(\mathrm{~d})$ shows the same fitting process to $d I / d V-z$ curves and $\kappa / \kappa_{N}$ extracted from the fit. Here, there is no divergence of fit $\sim 0 \mathrm{mV}$ because of nonzero $d I / d V$ at zero bias. However, overall $R^{2}$ is smaller for $d I / d V-z$ fitting than the $I-z$ fitting due to the better signal-to-noise ratio of $I$.

\section{APPENDIX C: JOSEPHSON CRITICAL CURRENT FROM THE $P(E)$ THEORY AND COMPARISON WITH THE AB FORMULA}

To get more convincing evidence that the $I-V$ and $d I / d V$ curves around zero bias are truly from the Josephson tunneling, we tried to extract Josephson critical current $I_{0}$ by fitting the $I-V$ curves to the $P(E)$ theory and compare with the $\mathrm{AB}$ formula. To extract $I_{0}$, we followed Jäck et al. [15] and used $P(E)$ theory that derives Josephson tunneling current as

$$
I(V)=\frac{\pi e}{\hbar} E_{J}^{2}[P(2 e V)-P(-2 e V)],
$$

where $P(E)$ is the tunneling probability of a Cooper pair, and $E_{J}$ is Josephson energy that can be expressed as $E_{J}=\frac{\hbar}{2 e} I_{0}$. The AB formula predicts $I_{0}$ to be only a function of normal conductance $G_{N}$ and superconducting gaps $\Delta$ as

$$
I_{0}=\Delta_{2} G_{N} K\left(\sqrt{1-\frac{\Delta_{2}^{2}}{\Delta_{1}^{2}}}\right), \quad\left(\Delta_{1}>\Delta_{2}\right) .
$$

Figure 7(a) shows the fitting of $I-V$ curves to the $P(E)$ theory, and Fig. 7(b) shows the $I_{0}$ vs $G_{N} / G_{0}$ from the fitting and $\mathrm{AB}$ formula. We note that our $I-V$ curves do not show the pronounced peak-and-dip feature as in Jäck et al. [15] and other references [12,14,16,17] due to the high effective measurement temperature of $3.2 \mathrm{~K}$ compared with most other experiments done at the temperature of $1 \mathrm{~K}$ or less. Still, the $I_{0}$ from the $P(E)$ fitting shows a perfect match to the AB formula, which is strong evidence that the tunneling current around zero bias is from the Josephson effect.

\section{APPENDIX D: MAPS AND HISTOGRAMS OF $\kappa / \kappa_{N}$ FOR FULL BIAS RANGE}

Movie S1 in the Supplemental Material [31] displays all $\kappa / \kappa_{N}$ maps for full bias range (left) and the probability density of $\kappa / \kappa_{N}$ from their histograms (right). (a)

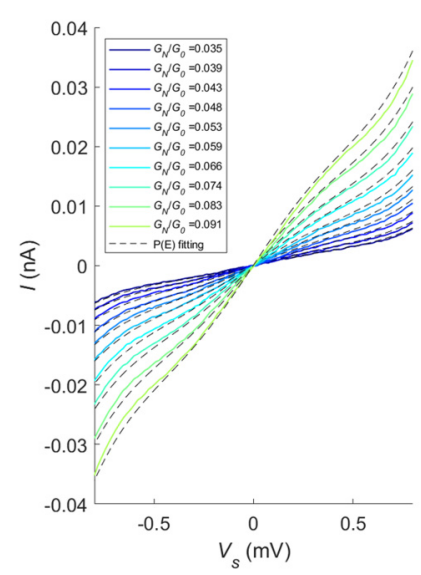

(b)

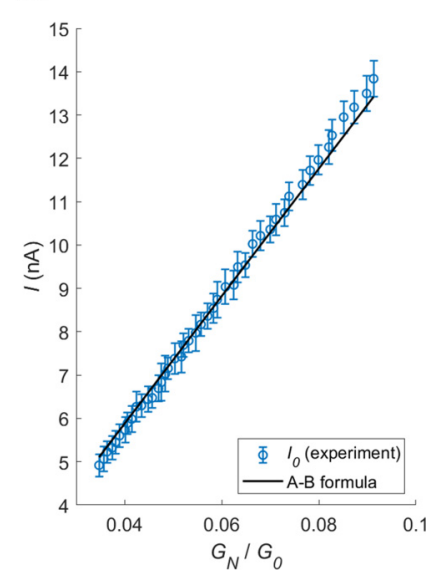

FIG. 7. (a) Fitting of $I-V$ curves in Fig. 2(a) with $P(E)$ theory. Note that the plot range of $I-V$ curves are zoomed in from Fig. 2(a) to make curves visible. (b) Comparison of Josephson critical current from the fitting in (a) and Ambegaokar-Baratoff (AB) formula. 
[1] B. D. Josephson, Possible new effects in superconductive tunnelling, Phys. Lett. 1, 251 (1962).

[2] M. Kjaergaard, M. E. Schwartz, J. Braumüller, P. Krantz, J. I. J. Wang, S. Gustavsson, and W. D. Oliver, Superconducting qubits: current state of play, Annu. Rev. Condens. Matter Phys. 11, 369 (2020).

[3] P. Krantz, M. Kjaergaard, F. Yan, T. P. Orlando, S. Gustavsson, and W. D. Oliver, A quantum engineer's guide to superconducting qubits, Appl. Phys. Rev. 6, 021318 (2019).

[4] T. Bauch, T. Lindström, F. Tafuri, G. Rotoli, P. Delsing, T. Claeson, and F. Lombardi, Quantum dynamics of a $d$-wave Josephson junction, Science 311, 57 (2006).

[5] J. F. Liu, Y. Xu, and J. Wang, Identifying the chiral $d$-wave superconductivity by Josephson $\varphi_{0}$-states, Sci. Rep. 7, 43899 (2017).

[6] Y. Tanaka, T. Yokoyama, and N. Nagaosa, Manipulation of the Majorana Fermion, Andreev Reflection, and Josephson Current on Topological Insulators, Phys. Rev. Lett. 103, 107002 (2009).

[7] M. Tinkham, Introduction to Superconductivity, Second edition (Dover Publications, New York, 2004).

[8] V. Ambegaokar and A. Baratoff, Tunneling between Superconductors, Phys. Rev. Lett. 10, 486 (1963).

[9] S. Shapiro, Josephson Currents in Superconducting Tunneling: The effect of Microwaves and Other Observations, Phys. Rev. Lett. 11, 80 (1963).

[10] R. C. Dynes, V. Narayanamurti, and J. P. Garno, Direct Measurement of Quasiparticle-Lifetime Broadening in a Strong-Coupled Superconductor, Phys. Rev. Lett. 41, 1509 (1978).

[11] E. Scheer, N. Agraït, J. C. Cuevas, A. L. Yeyati, B. Ludoph, A. Martín-Rodero, G. R. Bollinger, J. M. Van Ruitenbeek, and C. Urbina, The signature of chemical valence in the electrical conduction through a single-atom contact, Nature 394, 154 (1998).

[12] O. Naaman, W. Teizer, and R. C. Dynes, Fluctuation Dominated Josephson Tunneling with a Scanning Tunneling Microscope, Phys. Rev. Lett. 87, 097004 (2001).

[13] J. Šmakov, I. Martin, and A. V. Balatsky, Josephson scanning tunneling microscopy, Phys. Rev. B 64, 212506 (2001).

[14] M. Ternes, W. D. Schneider, J. C. Cuevas, C. P. Lutz, C. F. Hirjibehedin, and A. J. Heinrich, Subgap structure in asymmetric superconducting tunnel junctions, Phys. Rev. B 74, 132501 (2006).

[15] B. Jäck, M. Eltschka, M. Assig, M. Etzkorn, C. R. Ast, and K. Kern, Critical Josephson current in the dynamical Coulomb blockade regime, Phys. Rev. B 93, 020504(R) (2016).

[16] M. T. Randeria, B. E. Feldman, I. K. Drozdov, and A. Yazdani, Scanning Josephson spectroscopy on the atomic scale, Phys. Rev. B 93, 161115(R) (2016).

[17] D. Cho, K. M. Bastiaans, D. Chatzopoulos, G. D. Gu, and M. P. Allan, A strongly inhomogeneous superfluid in an iron-based superconductor, Nature 571, 541 (2019).

[18] M. Ruby, B. W. Heinrich, J. I. Pascual, and K. J. Franke, Experimental Demonstration of a Two-Band Superconducting State for Lead Using Scanning Tunneling Spectroscopy, Phys. Rev. Lett. 114, 157001 (2015).

[19] K. J. Franke, G. Schulze, and J. I. Pascual, Competition of superconducting phenomena and Kondo screening at the nanoscale, Science 332, 940 (2011).
[20] J. Bardeen, Tunnelling from a Many-Particle Point of View, Phys. Rev. Lett. 6, 57 (1961).

[21] J. Tersoff and D. R. Hamann, Theory and Application for the Scanning Tunneling Microscope, Phys. Rev. Lett. 50, 1998 (1983).

[22] G. Johansson, P. Samuelsson, and Å. Ingerman, Full Counting Statistics of Multiple Andreev Reflection, Phys. Rev. Lett. 91, 187002 (2003).

[23] J. C. Cuevas and W. Belzig, Full Counting Statistics of Multiple Andreev Reflections, Phys. Rev. Lett. 91, 187001 (2003).

[24] J. C. Cuevas, A. Martín-Rodero, and A. L. Yeyati, Hamiltonian approach to the transport properties of superconducting quantum point contacts, Phys. Rev. B 54, 7366 (1996).

[25] M. H. Devoret, D. Esteve, H. Grabert, G. L. Ingold, H. Pothier, and C. Urbina, Effect of the Electromagnetic Environment on the Coulomb Blockade in Ultrasmall Tunnel Junctions, Phys. Rev. Lett. 64, 1824 (1990).

[26] G. L. Ingold, H. Grabert, and U. Eberhardt, Cooper-pair current through ultrasmall Josephson junctions, Phys. Rev. B 50, 395 (1994).

[27] C. R. Ast, B. Jäck, J. Senkpiel, M. Eltschka, M. Etzkorn, J. Ankerhold, and K. Kern, Sensing the quantum limit in scanning tunnelling spectroscopy, Nat. Commun. 7, 13009 (2016).

[28] G. E. Blonder, M. Tinkham, and T. M. Klapwijk, Transition from metallic to tunneling regimes in superconducting microconstrictions: excess current, charge imbalance, and supercurrent conversion, Phys. Rev. B 25, 4515 (1982).

[29] J. Brand, P. Ribeiro, N. Néel, S. Kirchner, and J. Kröger, Impact of Atomic-Scale Contact Geometry on Andreev Reflection, Phys. Rev. Lett. 118, 107001 (2017).

[30] K. M. Bastiaans, D. Cho, D. Chatzopoulos, M. Leeuwenhoek, C. Koks, and M. P. Allan, Imaging doubled shot noise in a Josephson scanning tunneling microscope, Phys. Rev. B 100, 104506 (2019).

[31] See Supplemental Material at http://link.aps.org/supplemental/ 10.1103/PhysRevResearch.3.033248 for full dataset of the relative decay constant maps.

[32] S. Kullback and R. A. Leibler, On information and sufficiency, Ann. Math. Statist. 22, 79 (1951).

[33] C. W. Groth, M. Wimmer, A. R. Akhmerov, and X. Waintal, KWANT: a software package for quantum transport, New J. Phys. 16, 063065 (2014).

[34] Z. Du, H. Li, S. H. Joo, E. P. Donoway, J. Lee, J. C. S. Davis, G. Gu, P. D. Johnson, and K. Fujita, Imaging the energy gap modulations of the cuprate pair-density-wave state, Nature $\mathbf{5 8 0}$, 65 (2020).

[35] M. H. Hamidian, S. D. Edkins, S. H. Joo, A. Kostin, H. Eisaki, S. Uchida, M. J. Lawler, E.-A. Kim, A. P. Mackenzie, K. Fujita, J. Lee, and J. C. Séamus Davis, Detection of a Cooper-pair density wave in $\mathrm{Bi}_{2} \mathrm{Sr}_{2} \mathrm{CaCu}_{2} \mathrm{O}_{8+x}$, Nature 532, 343 (2016).

[36] K. T. Law, P. A. Lee, and T. K. Ng, Majorana Fermion Induced Resonant Andreev Reflection, Phys. Rev. Lett. 103, 237001 (2009).

[37] S. Zhu, L. Kong, L. Cao, H. Chen, M. Papaj, S. Du, Y. Xing, W. Liu, D. Wang, C. Shen, F. Yang, J. Schneeloch, R. Zhong, G. Gu, L. Fu, Y.-Y. Zhang, H. Ding, and H.-J. Gao, Nearly quantized conductance plateau of vortex zero mode in an iron-based superconductor, Science 367, 189 (2020).

[38] http://energy.gov/downloads/doe-public-access-plan. 